54, 2, pp. 621-632, Warsaw 2016

DOI: $10.15632 /$ jtam-pl.54.2.621

\title{
INVESTIGATION ON A QUASI-ZERO-STIFFNESS VIBRATION ISOLATOR UNDER RANDOM EXCITATION
}

\author{
Yong Wang, Shunming Li, Chun Cheng \\ College of Energy and Power Engineering, Nanjing University of Aeronautics and Astronautics, Nanjing, China \\ e-mail:wangy1921@126.com
}

\begin{abstract}
The dynamic response and isolation performance of a Quasi-Zero-Stiffness (QZS) vibration isolator using inclined springs as negative stiffness correctors under random excitation are presented in this paper. The probabilistic linearization method is employed to determine the dynamic response of the QZS vibration isolator and compared with the exact solution based on the Fokker-Planck-Kolmogorov (FPK) equation and the equivalent linearization method. Two performance indexes (Mean Square Relative Displacement (MSRD) and Mean Square Acceleration (MSA)) are considered to evaluate the isolation performance of the QZS vibration isolator under random excitation and compared with the equivalent linear vibration isolator. The results show that the MSRD of the QZS vibration isolator is always lower than the equivalent linear vibration isolator, while the MSA of the QZS vibration isolator can be larger or lower than the equivalent linear vibration isolator based on the values of damping ratio and spectral density of the random excitation.
\end{abstract}

Keywords: vibration isolator, quasi-zero-stiffness, random excitation, dynamic analysis, performance analysis

\section{Introduction}

Nonlinear vibration isolators with Quasi-Zero-Stiffness (QZS) characteristic (Ibrahim, 2008) have been developed to improve the vibration isolation performance of passive linear vibration isolators and have drawn much attention in the engineering industry since they can provide lower vibration isolation frequency without sacrificing the load bearing capacity. The QZS vibration isolator usually comprises of a load bearing elastic element providing positive stiffness and special mechanisms providing negative stiffness named as negative stiffness correctors. Alabuzhev et al. (1989) investigated the effect of negative stiffness correctors and summarized many prototypes of QZS vibration isolators. Carrella et al. (2007), Kovacic et al. (2008) and Hao and Cao et al. (2014) considered a QZS vibration isolator by using inclined springs as negative stiffness correctors and studied the static and dynamic characteristics theoretically. Le and Ahn (2011) built a QZS vibration isolator composed of a positive stiffness mount and two symmetric negative stiffness structures for improving vibration isolation performance of the vehicle seat. Robertson et al. (2009), Zhou and Liu (2010) and Xu et al. (2013) used electromagnetic springs or magnetic springs as negative stiffness correctors to build a QZS vibration isolator and studied the static and dynamic characteristics detailedly. Liu et al. (2013) designed a QZS vibration isolator by using Euler buckled beams as negative stiffness correctors and analyzed the dynamic behavior theoretically. Shaw et al. (2013) used bistable composite plates as negative stiffness correctors to form a QZS vibration isolator and investigated the dynamic response theoretically and experimentally.

In most of the above mentioned researches, the dynamic response and vibration isolation performance of the QZS vibration isolators under harmonic excitation have been investigated in detail. The QZS vibration isolator can also endure shock excitation or random excitation, which 
are common in the practical engineering. Liu et al. (2013) and Wang et al. (2014) considered the performance of the QZS vibration isolators under shock excitation systematically. But the performance of the QZS vibration isolator subjected to random excitation has been rarely discussed in detail. Linear vibration isolators under random excitation were studied fully by Harris and Piersol (2002). Lyon $(1960,1961)$ investigated vibration statistics of a randomly excited hardspring oscillator and obtained an expression of the joint density of displacement and velocity. Klein (1964) considered the random excitation of a nonlinear system with tangent elasticity characteristics and studied the dynamic behavior of the nonlinear system in detail. Kirk (1988) compared dynamic performances of three different kinds of nonlinear vibration isolators with cubic hard, cubic soft and tangent stiffness comprehensively. Shin (2014) did experimental investigation of the vibration transmissibility of a magnet-spring vibration isolator under random excitation.

A number of approximated analytic methods have been developed to study the dynamic response of nonlinear vibration isolators under random excitation, such as the method based on the Fokker-Planck-Kolmogorov (FPK) equation (Lin, 1967), equivalent linearization method (Caughey, 1963), partial linearization method (Elishakoff and Cai, 1993), dissipation energy balancing and weighted residuals method (Cai and Lin, 1988), and cumulant-neglect closure method (Wu and Lin, 1984). In this paper, the probabilistic linearization method (Polidori and Beck, 1996; Polidori et al., 2000) is used. The probabilistic linearization method finds a linear vibration system which best approximates the true nonlinear vibration system and minimizes the error of the FPK equation rather than the stochastic differential equation, it can yield simple expressions to determine the desired probabilistic characteristics of the dynamic response of the nonlinear vibration system.

The organization of this paper is as follows. A QZS vibration isolator using inclined springs as negative stiffness correctors (Carrella et al., 2007) is presented and a brief static analysis of the QZS vibration isolator is shown in Section 2. In Section 3, a brief description of the probabilistic linearization method is introduced, the dynamic response of the QZS vibration isolator under random excitation using this method is obtained and compared with the exact solution based on the FPK equation and the equivalent linearization method. In Section 4, two performance indexes are considered to evaluate the isolation performance of the QZS vibration isolator and compared with an equivalent linear vibration isolator. Conclusions are drawn in Section 5.

\section{Static analysis of a QZS vibration isolator}

A QZS vibration isolator comprised of a vertical spring used as the load bearing element and inclined springs used as negative stiffness correctors is shown in Fig. 1. Figure 1 also shows when loading a mass $m$, the system is balanced at the static equilibrium position, and the inclined springs are in the horizontal position. The stiffness of the vertical and inclined springs are $K_{v}$ and $K_{h}$; the initial length of the inclined springs is $l_{0}$ and the length when they are in the horizontal position is $l$; the damping coefficient of the damper is $c ; x$ is the displacement of the mass from the static equilibrium position and $y$ is the base excitation with random input.

The force-displacement and stiffness-displacement relationships of the QZS vibration isolator are given as

$$
F=K_{v}(x-y)+2 K_{h}\left(1-\frac{l_{0}}{\sqrt{x^{2}+l^{2}}}\right)(x-y) \quad K=K_{v}+2 K_{h}-\frac{2 K_{h} l_{0} l^{2}}{\sqrt{\left[(x-y)^{2}+l^{2}\right]^{3}}}
$$

Equation (2.1) can be written in non-dimensional form as

$$
\widehat{F}=z+2 k\left(1-\frac{1}{\sqrt{z^{2}\left(1-\widehat{l}^{2}\right)+\widehat{l}^{2}}}\right) z \quad \widehat{K}=1+2 k-\frac{2 k \widehat{l}^{2}}{\sqrt{\left[z^{2}\left(1-\widehat{l}^{2}\right)+\widehat{l}^{2}\right]^{3}}}
$$




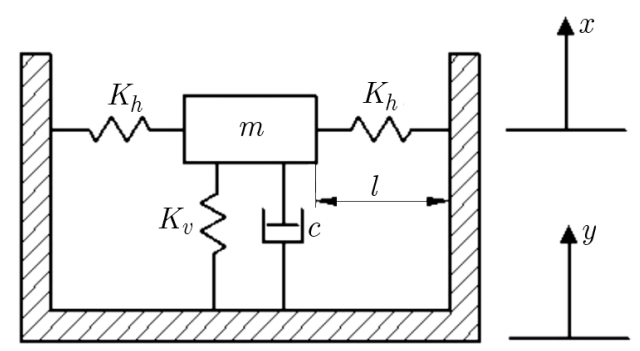

Fig. 1. Model of a QZS vibration isolator

where $z=(x-y) / x_{s}, \widehat{F}=F /\left(K_{v} x_{s}\right), \widehat{l}=l / l_{0}, k=K_{h} / K_{v}, \widehat{K}=K / K_{v}, x_{s}=\sqrt{l_{0}^{2}-l^{2}}$ is the static equilibrium displacement.

The non-dimensional stiffness of the QZS vibration isolator at the static equilibrium position can be obtained by substituting $z=0$ into Eq. $(2.2)_{2}$

$$
\widehat{K}_{s}=1+2 k\left(1-\frac{1}{\widehat{l}}\right)
$$

If the stiffness of the QZS vibration isolator is zero at the static equilibrium position, the QZS characteristic can be achieved and then the value of $\widehat{l}$ is given as

$$
\widehat{l}_{Q Z S}=\frac{2 k}{1+2 k}
$$

The non-dimensional force-displacement and stiffness-displacement curves of the QZS vibration isolator for various values of $\hat{l}$ when $k=1$ are shown in Fig. 2. It can be seen that when $\widehat{l}=\widehat{l}_{Q Z S}$, the positive stiffness of the vertical spring is balanced by the negative stiffness provided by the inclined springs at the static equilibrium position, then the QZS characteristic can be achieved. When $\hat{l}<\widehat{l}_{Q Z S}$, the stiffness of the QZS vibration isolator is negative in the neighborhood of the static equilibrium position which is an undesirable condition in the engineering practice. When $\widehat{l}>\widehat{l}_{Q Z S}$, the stiffness of the QZS vibration isolator maintains a small positive value at the static equilibrium position. So in order to keep the stiffness positive, $\hat{l}$ should be greater than or equal to $\widehat{l}_{Q Z S}$.
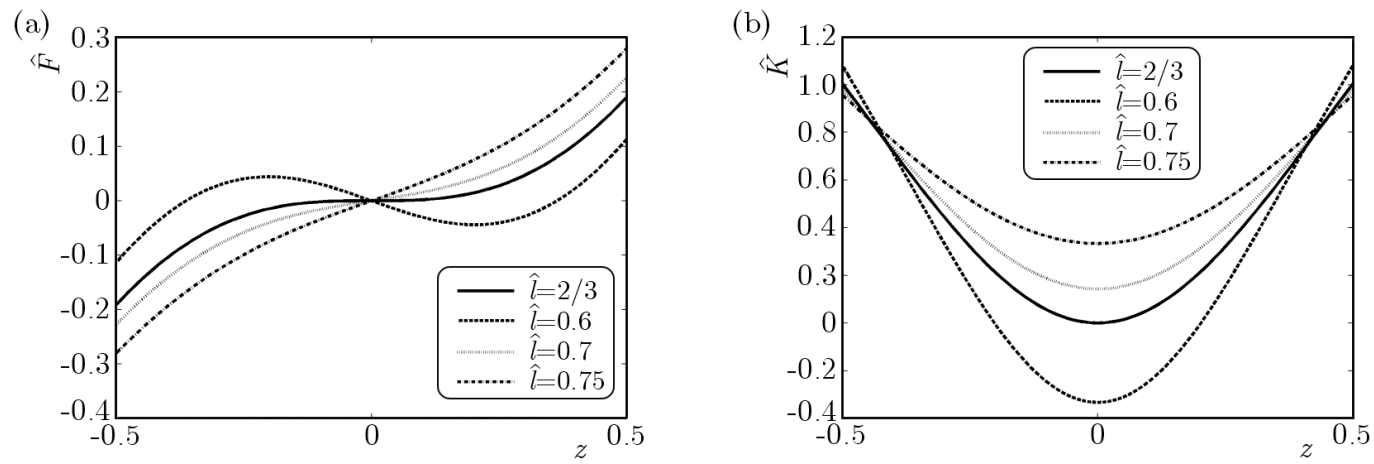

Fig. 2. Non-dimensional force-displacement and stiffness-displacement curves

When the amplitude of the displacement is small, the non-dimensional force and stiffness can be expanded as a Taylor series at the static equilibrium position $z=0$ for simplicity

$$
\begin{array}{ll}
\widehat{F}_{a}(z)=\left(1-2 k \frac{1-\widehat{l}}{\widehat{l}}\right) z+k \frac{1-\widehat{l}^{2}}{\widehat{l}^{3}} z^{3}=\alpha z+\gamma z^{3} & \widehat{K}_{a}(\widehat{x})=\alpha+3 \gamma z^{2} \\
\alpha=1-2 k \frac{1-\widehat{l}}{\widehat{l}} & \gamma=k \frac{1-\widehat{l}^{2}}{\widehat{l}^{3}}
\end{array}
$$


The dynamic equation of the QZS vibration isolator under random base excitation using a third-order Taylor series expansion is given as

$$
m \ddot{x}+c(\dot{x}-\dot{y})+\alpha K_{v}(x-y)+\gamma K_{v} \frac{(x-y)^{3}}{x_{s}^{2}}=0
$$

where the dots denote derivatives with respect to time $t$. Equation (2.6) can be written in non-dimensional form as

$$
z^{\prime \prime}+2 \zeta z^{\prime}+z+\lambda z^{3}=f(T)
$$

where $\zeta=c /\left(2 m w_{n}\right), \lambda=\gamma / \alpha, w_{n}=\sqrt{\alpha K_{v} / m}, T=w_{n} t, f(T)=-y^{\prime \prime} / x_{s}$. The primes denote derivatives with respect to $T$. When the base excitation is random excitation, the mathematical expression of the non-dimensional function $f(T)$ can be expressed as

$$
f(T)=\sqrt{S_{0}} n(T)
$$

where $n(T)$ is a stationary zero-mean Gaussian white noise with $E[n(T) n(T+\tau)]=\delta(\tau)$ and $S_{0}$ is the spectral density. Then, Eq. (2.7) can be written as

$$
z^{\prime \prime}+2 \zeta z^{\prime}+z+\lambda z^{3}=\sqrt{S_{0}} n(T)
$$

\section{Response of the QZS vibration isolator under random excitation}

The response of the QZS vibration isolator under random excitation is obtained by using the probabilistic linearization method. The probabilistic linearization method finds an equivalent linear vibration system whose stationary probability density function best fits the FPK equation of the nonlinear vibration system. Consider the Itô stochastic differential equation

$$
d z(T)=g(z, T) d T+h(z, T) d w(T)
$$

where $z \in R^{n}, g(z, T) \in R^{n}, h(z, T) \in R^{n \times m}$ and $w(T) \in R^{m}$ is a normalized Wiener process with $E\left[\left(w_{i}\left(T_{1}\right)-w_{i}\left(T_{2}\right)\right)\left(w_{j}\left(T_{1}\right)-w_{j}\left(T_{2}\right)\right)\right]=\left|T_{1}-T_{2}\right| \delta_{i j}$. The FPK equation is a linear equation governing the evolution of the state transition probability density function $p\left(z, T \mid z_{0}, T_{0}\right)$ of the system, which is given as

$$
\frac{\partial}{\partial T} p\left(z, T \mid z_{0}, T_{0}\right)=L(z, T) p\left(z, T \mid z_{0}, T_{0}\right)
$$

where $L(z, T)$ is the forward Kolmogorov operator expressed as

$$
L(z, T) \varphi(z, T)=-\sum_{i=1}^{n} \frac{\partial}{\partial z_{i}}\left(g_{i}(z, T) \varphi(z, T)\right)+\frac{1}{2} \sum_{i=1}^{n} \sum_{j=1}^{n} \frac{\partial}{\partial z_{i}} \frac{\partial}{\partial z_{j}}\left(b_{i j}(z, T) \varphi(z, T)\right)
$$

where $b(z, T)=h(z, T) h^{T}(z, T) \in R^{n \times n}$. The equivalent linear vibration system used to approximate Eq. (3.1) is defined as

$$
d z(T)=A_{e q}(\sigma) z(T) d T+B_{e q}(\sigma) d w(T)
$$

where $A_{e q}(\sigma)$ and $B_{e q}(\sigma)$ are the matrices of appropriate dimensions and $\sigma$ is a parameter vector of the equivalent linear vibration system. 
Define $L_{n l}(z)$ and $L_{l i n}(z \mid \sigma)$ as the forward Kolmogorov operators of the nonlinear vibration system and equivalent linear vibration system respectively. Let $p_{n l}(z)$ and $p_{l i n}(z \mid \sigma)$ be stationary solutions of their corresponding FPK equations, then gives

$$
L_{n l}(z) p_{n l}(z)=0 \quad L_{l i n}(z \mid \sigma) p_{l i n}(z \mid \sigma)=0
$$

where $p_{l i n}(z \mid \sigma)$ is the Gaussian probability density function of the equivalent linear vibration system and depends on the parameter vector $\sigma$. The main objective of the probabilistic linearization method is to find a probability density $p_{l i n}(z \mid \sigma)$ to satisfy the following condition

$$
L_{n l}(z) p_{l i n}(z \mid \sigma) \approx 0
$$

The criterion for making this condition is chosen as

$$
\min _{\sigma}\left\|L_{n l}(z) p_{l i n}(z \mid \sigma)\right\|
$$

where the norm is a standard or weighted $\Re^{2}$ norm. For any two functions: $f(z), g(z): R^{n} \rightarrow R$, the standard $\Re^{2}$ inner product of these two functions is defined as

$$
\langle f, g\rangle=\int_{R^{n}} f(z) g(z) d z
$$

For any weighted function $\mu(z)>0$, a weighted inner product of these two functions is defined as

$$
\langle f, g\rangle_{\mu}=\int_{R^{n}} f(z) g(z) \mu(z) d z
$$

So a standard $\Re^{2}$ norm and weighted $\Re^{2}$ norm of the criterion can be obtained

$$
\begin{aligned}
& \left\|L_{n l}(z) p_{l i n}(z \mid \sigma)\right\|_{\Re^{2}}^{2}=\int_{R^{n}}\left(L_{n l}(z) p_{l i n}(z \mid \sigma)\right)^{2} d z \\
& \left\|L_{n l}(z) p_{l i n}(z \mid \sigma)\right\|_{\Re^{2}(\mu)}^{2}=\int_{R^{n}}\left(L_{n l}(z) p_{l i n}(z \mid \sigma)\right)^{2} \mu(z) d z
\end{aligned}
$$

The weighted function $\mu(z)$ is chosen to put emphasis in the approximations to the tails of the probability density function of the nonlinear vibration system, which is known to be non-Gaussian for the nonlinear vibration system.

The exact probability density function of the nonlinear vibration system based on the FPK equation expressed by Eq. (2.9) is given by

$$
p\left(z_{1}, z_{2}\right)=\frac{\sqrt{4 \zeta \lambda /\left(\pi S_{0}\right)}}{\mathrm{e}^{\varepsilon} K_{1 / 4}(\varepsilon)} \exp \left[-\frac{4 \zeta}{S_{0}}\left(\frac{1}{2} z_{1}^{2}+\frac{1}{4} \lambda z_{1}^{2}+\frac{1}{2} z_{2}^{2}\right)\right]
$$

where $z_{1}=z, z_{2}=z^{\prime}, \varepsilon=\zeta /\left(2 \lambda S_{0}\right)$ and $K_{1 / 4}$ is a modified Bessel function of the second kind.

Rewriting Eq. (2.9) in form of Eq. (3.1), gives

$$
\left[\begin{array}{l}
d z_{1}(T) \\
d z_{2}(T)
\end{array}\right]=\left[\begin{array}{c}
z_{2} \\
-2 \zeta z_{2}-z_{1}-r z_{1}^{3}
\end{array}\right] d T+\left[\begin{array}{c}
0 \\
\sqrt{S_{0}}
\end{array}\right] d w(T)
$$

The associated equivalent linear vibration system is obtained as

$$
\left[\begin{array}{l}
d z_{1}(T) \\
d z_{2}(T)
\end{array}\right]=\left[\begin{array}{cc}
0 & 1 \\
-w_{e q}^{2} & -2 \zeta_{e q}
\end{array}\right]\left[\begin{array}{l}
z_{1} \\
z_{2}
\end{array}\right] d T+\left[\begin{array}{c}
0 \\
\sqrt{S_{0}}
\end{array}\right] d w(T)
$$


The stationary probability density function of the equivalent linear vibration system can be obtained as the following two terms

$$
\begin{aligned}
& p_{\text {lin }}\left(z \mid w_{w q}^{2}, \zeta_{e q}\right)=\frac{4 \zeta_{e q} w_{e q}}{2 \pi S_{0}} \exp \left[-\left(\frac{2 \zeta_{e q} w_{e q}^{2}}{S_{0}} z_{1}^{2}+\frac{2 \zeta_{e q}}{S_{0}} z_{2}^{2}\right)\right] \\
& p_{l i n}\left(z \mid \sigma, \sigma_{z_{2}}\right)=\frac{1}{2 \pi \sigma \sigma_{z_{2}}} \exp \left[-\left(\frac{z_{1}^{2}}{2 \sigma^{2}}+\frac{z_{2}^{2}}{2 \sigma_{z_{2}}^{2}}\right)\right]
\end{aligned}
$$

Since the damping ratio of the nonlinear vibration system is linear, the probability density function of $p_{n l}\left(z_{2}\right)$ is a Gaussian probability density function, then Eq. (3.14) 2 can be obtained as a function of the parameter $\sigma$

$$
p_{\text {lin }}(z \mid \sigma)=\frac{1}{2 \pi \sigma \sigma_{z_{2}}} \exp \left[-\left(\frac{z_{1}^{2}}{2 \sigma^{2}}+\frac{z_{2}^{2}}{2 \sigma_{z_{2}}^{2}}\right)\right]
$$

where $\sigma_{z_{2}}^{2}=S_{0} /(4 \zeta)$. The forward Kolmogorov operators of the nonlinear vibration system and the probabilistic linearization method criterion are given by

$$
\begin{aligned}
& L_{n l}(z) p(z)=\frac{S_{0}}{2} \frac{\partial^{2} p(z)}{\partial z_{2}^{2}}-\frac{\partial}{\partial z_{1}}\left[z_{2} p(z)\right]+\frac{\partial}{\partial z_{2}}\left[\left(2 \zeta z_{2}+z_{1}+\lambda z_{1}^{3}\right) p(z)\right] \\
& \min _{\sigma}\left\|L_{n l}(z) p_{l i n}(z \mid \sigma)\right\|
\end{aligned}
$$

Using the standard $\Re^{2}$ norm, Eqs. (3.16) can be written as

$$
\begin{aligned}
& L_{n l}(z) p_{l i n}(z \mid \sigma)=\left[\left(\frac{S_{0}}{2 \sigma_{z_{2}}^{4}}-\frac{2 \zeta}{\sigma_{z_{2}}^{2}}\right) z_{2}^{2}+\left(\frac{z_{1}}{\sigma_{z_{1}}^{2}}-\frac{z_{1}}{\sigma_{z_{2}}^{2}}-\frac{\lambda z_{1}^{3}}{\sigma_{z_{2}}^{2}}\right) z_{2}+2 \zeta-\frac{S_{0}}{2 \sigma_{z_{2}}^{2}}\right) p_{\text {lin }}(z \mid \sigma) \\
& \min _{\sigma}\left\|L_{n l}(z) p_{l i n}(z \mid \sigma)\right\|_{\Re^{2}}^{2}=\frac{15 \lambda^{2}}{64 \pi \sigma_{z_{2}}^{3}} \sigma^{5}+\frac{3 \lambda}{16 \pi \sigma_{z_{2}}^{3}} \sigma^{3}+\frac{1}{16 \pi}\left(-\frac{3 \lambda}{\sigma_{z_{2}}}+\frac{1}{\sigma_{z_{2}}^{3}}\right) \sigma \\
& \quad+\left[\left(\frac{3}{4 \pi} \zeta^{2}-\frac{1}{8 \pi}\right) \frac{1}{\sigma_{z_{2}}}-\frac{3 \zeta S_{0}}{8 \pi \sigma_{z_{2}}^{3}}+\frac{3 S_{0}^{2}}{64 \pi \sigma_{z_{2}}^{5}}\right] \frac{1}{\sigma}+\frac{\sigma_{z_{2}}}{16 \pi \sigma^{3}}
\end{aligned}
$$

To minimize the criterion, let $\partial \min _{\sigma}\left\|L_{n l}(z) p_{l i n}(z \mid \sigma)\right\|_{\Re^{2}}^{2} / \partial \sigma=0$, then $\sigma$ can be obtained numerically.

$\sigma_{z}$ can also be obtained by using the equivalent linearization method, then it gives

$$
\sigma_{z}^{2}=\frac{\sqrt{\zeta^{2}+3 \zeta \lambda S_{0}}-\zeta}{6 \zeta \lambda}
$$

Figure 3 shows the probability density function $p(z)$ of the QZS vibration isolator using different analytical methods. It can be seen that both the probabilistic linearization method and equivalent linearization method give good results in the tails of the probability density function, but the errors become larger in the peak value areas of the probability density function and the probabilistic linearization method gives better results than the equivalent linearization method. When $\hat{l}$ increases, the parameter $\alpha$ increases and $\gamma$ decreases, which indicates that the nonlinear parameter $\lambda$ becomes smaller, the errors of both methods become smaller in the peak value areas of the probability density function.

\section{Performance of the QZS vibration isolator under random excitation}

The performance of the QZS vibration isolator under random excitation is evaluated by two performance indexes, which are defined as follows: 

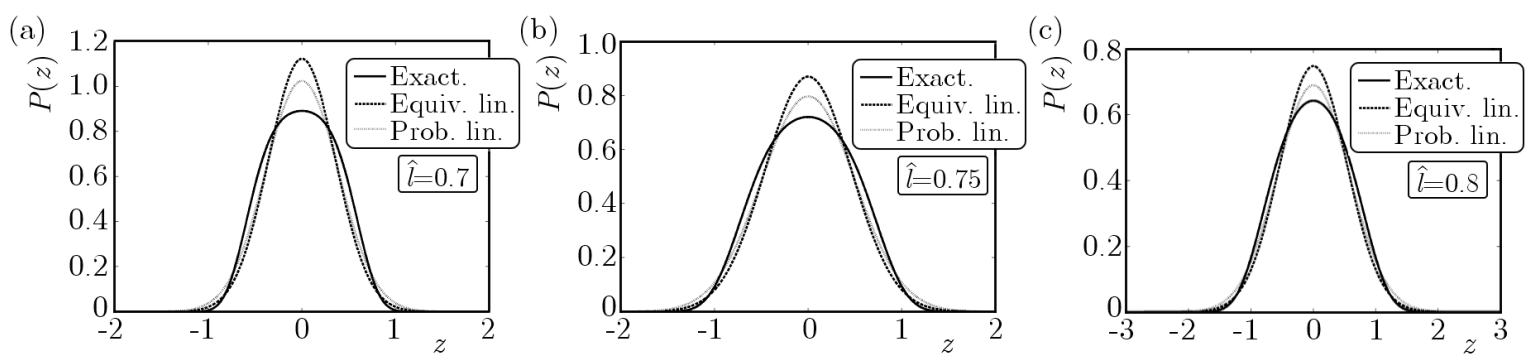

Fig. 3. Probability density function $p(z)$ of the QZS vibration isolator using different analytic methods $\left(\zeta=0.02, S_{0}=0.05\right)$

(1) Mean square relative displacement (MSRD) $E\left(z^{2}\right)$,

(2) Mean square acceleration (MSA) $E\left(z^{\prime \prime 2}\right)$.

It is also of interest to compare the isolation performance of the QZS vibration isolator with an equivalent linear vibration isolator with the same load bearing capacity. Since the QZS vibration isolator is comprised of the load bearing element and negative stiffness correctors, the equivalent linear vibration isolator is the QZS vibration isolator with the negative stiffness correctors removed, then the natural frequency and damping ratio of the equivalent linear vibration isolator can be obtained

$$
w_{l}=\frac{w_{n}}{\sqrt{\alpha}} \quad \zeta_{l}=\sqrt{\alpha} \zeta
$$

\subsection{Mean square relative displacement (MSRD)}

The MSRD can be obtained by using both the probabilistic linearization method and equivalent linearization method, which can be clearly seen in Eq. (3.17) and Eq. (3.18). The MSRD obtained by the probabilistic linearization method can be improved by using a weighted function. The weighted function $\mu(z)=1+z^{2}$ is used in calculating $E\left(z^{2}\right)$. Although the chosen weighted function can be arbitrary, this particular function is chosen for three reasons:

(1) Give more weight to the tails of the probability density function $p(z)$, as the probability density function $p(z)$ for small values of $z$ is not as important when calculating MSRD.

(2) The weighted function should not significantly increase the computation complexity.

(3) It seems reasonable to include a $z^{2}$ term in calculating $E\left(z^{2}\right)$. Then the weighted $\Re^{2}$ norm of the criterion combining Eq. (3.10) 2 and Eq. $(3.17)_{1}$ can be obtained

$$
\begin{aligned}
\min _{\sigma} & \left\|L_{n l}(z) p_{l i n}(z \mid \sigma)\right\|_{\Re^{2}(\mu)}^{2}=\frac{105 \lambda^{2}}{128 \pi \sigma_{z_{2}}^{3}} \sigma^{7}+\frac{15\left(\lambda^{2}+2 \lambda\right)}{64 \pi \sigma_{z_{2}}^{3}} \sigma^{5}+\frac{1}{32 \pi}\left(-\frac{15 \lambda}{\sigma_{z_{2}}}+\frac{3+6 \lambda}{\sigma_{z_{2}}^{3}}\right) \sigma^{3} \\
+ & {\left[\left(\frac{3}{8 \pi} \zeta^{2}-\frac{3 \lambda}{16 \pi}-\frac{3}{16 \pi}\right) \frac{1}{\sigma_{z_{2}}}+\left(\frac{1}{16 \pi}-\frac{3}{16 \pi} \zeta S_{0}\right) \frac{1}{\sigma_{z_{2}}^{3}}+\frac{3 S_{0}^{2}}{128 \pi \sigma_{z_{2}}^{5}}\right] \sigma } \\
+ & {\left[\frac{3 \sigma_{z_{2}}}{32 \pi}+\left(\frac{3}{4 \pi} \zeta^{2}-\frac{1}{8 \pi}\right) \frac{1}{\sigma_{z_{2}}}-\frac{3 \zeta S_{0}}{8 \pi \sigma_{z_{2}}^{3}}+\frac{3 S_{0}^{2}}{64 \pi \sigma_{z_{2}}^{5}}\right] \frac{1}{\sigma}+\frac{\sigma_{z_{2}}}{16 \pi \sigma^{3}} }
\end{aligned}
$$

To minimize the criterion, let $\partial \min _{\sigma}\left\|L_{n l}(z) p_{l i n}(z \mid \sigma)\right\|_{\Re^{2}(\mu)}^{2} / \partial \sigma=0$, then $\sigma$ can be obtained numerically.

Figure 4 shows the MSRD curves of the QZS vibration isolator using different analytical methods. The exact solutions can be determined by integrating Eq. (3.11) directly using the numerical method. It can be seen that the probabilistic linearization method overestimates the MSRD, while the equivalent linearization method underestimates it. The weighted probabilistic 


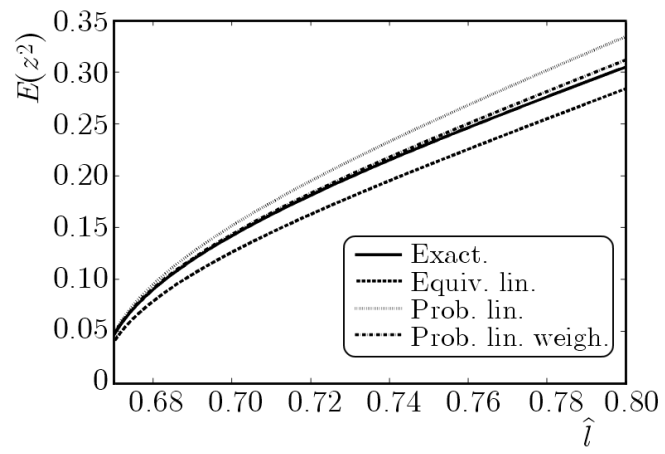

Fig. 4. MSRD curves of the QZS vibration isolator using different analytical methods

$$
\left(\zeta=0.02, S_{0}=0.05\right)
$$

linearization method gives better results with the numerical results than the other two analytical methods.

The MSRD curves of the QZS vibration isolator for different values of damping ratio and spectral density of the random excitation using the weighted probabilistic linearization method are shown in Fig. 5. The MSRD curves of the equivalent linear vibration isolator are also plotted in the same figure for comparison, which are plotted in the thinner lines. The MSRD of the equivalent linear vibration isolator is given as

$$
\sigma_{z_{l}}^{2}=\frac{S_{0}}{4 \zeta_{l}}=\frac{S_{0}}{4 \sqrt{\alpha} \zeta}
$$
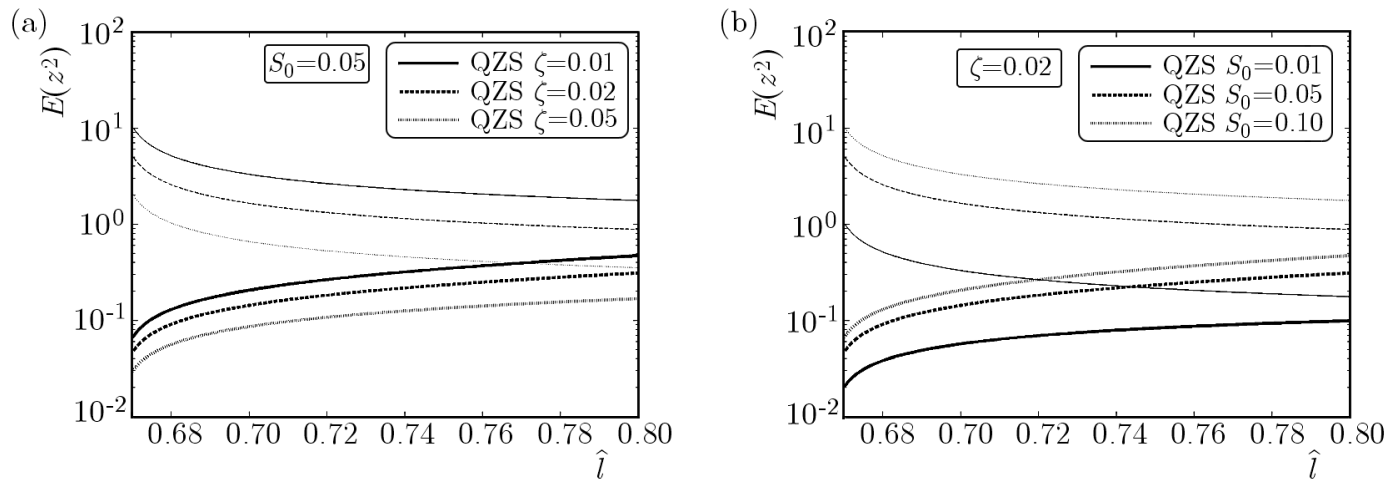

Fig. 5. MSRD curves of the QZS vibration isolator for different values of damping ratio and spectral density of the random excitation

The MSRD of the QZS vibration isolator is always lower than the equivalent linear vibration isolator, which indicates that the QZS vibration isolator can achieve a better isolation performance for the MSRD case. With an increase in the damping ratio $\zeta$ or a decrease in the spectral density $S_{0}$, the MSRD of both vibration isolators decreases. Also when the length ratio $\widehat{l}$ increases, the parameter $\alpha$ increases, $\gamma$ decreases and the nonlinear parameter $\lambda$ becomes smaller, then the MSRD of the equivalent linear vibration isolator decreases, while the MSRD of the QZS vibration isolator increases with an increase in the length ratio $\widehat{l}$.

\subsection{Mean square acceleration (MSA)}

The MSA is an important index for investigating the overall effects of various parameters on the response of the vibration isolator. The MSA can be obtained from Eq. (2.9)

$$
E\left(z^{\prime \prime 2}\right)=4 \zeta^{2} E\left(z^{\prime 2}\right)+E\left(z^{2}\right)+2 \gamma E\left(z^{4}\right)+\gamma^{2} E\left(z^{6}\right)
$$


The MSA can be determined by using both the probabilistic linearization method and equivalent linearization method. Using these two methods, Eq. (4.4) can be transformed as

$$
E\left(z^{\prime \prime 2}\right)=\zeta S_{0}+\sigma^{2}+6 \gamma \sigma^{4}+15 \gamma^{2} \sigma^{6}
$$

where $E\left(z^{2}\right)=\sigma^{2}$ is determined differently for these two methods. The MSA obtained by the probabilistic linearization method can be improved by using a weighted function. In this case, the weighted function $\mu(z)=1+\left(x_{1} z+x_{2} z^{3}\right)^{2}$ is chosen in calculating $E\left(z^{\prime \prime 2}\right)$, where $x_{1}$ and $x_{2}$ are larger values in order to put more weight to the tails of the probability density function because of the $\sigma^{2}, \sigma^{4}$ and $\sigma^{6}$ terms exist when it determines $E\left(z^{\prime \prime 2}\right)$. Then the weighted $\Re^{2}$ norm of the criterion can be obtained

$$
\begin{aligned}
\min _{\sigma}\left\|L_{n l}(z) p_{l i n}(z \mid \sigma)\right\|_{\Re^{2}(\mu)}^{2}=\frac{10395 \lambda^{2} x_{2}^{2}}{512 \pi \sigma_{z_{2}}^{3}} \sigma^{11}+\frac{945\left(\lambda^{2} x_{1} x_{2}+\lambda x_{2}^{2}\right)}{128 \pi \sigma_{z_{2}}^{3}} \sigma^{9} \\
+\frac{105}{128 \pi}\left(-\frac{9 \lambda x_{2}^{2}}{\sigma_{z_{2}}}+\frac{\lambda^{2} x_{1}^{2}+4 \lambda x_{1} x_{2}+x_{2}^{2}}{\sigma_{z_{2}}^{3}}\right) \sigma^{7}+\frac{15}{64 \pi}\left[\left(6 x_{2}^{2} \zeta^{2}-14 \lambda x_{1} x_{2}-7 x_{2}^{2}\right) \frac{1}{\sigma_{z_{2}}}\right. \\
\left.+\left(-3 x_{2}^{2} \zeta S_{0}+\lambda^{2}+2 \lambda x_{1}^{2}+2 x_{1} x_{2}\right) \frac{1}{\sigma_{z_{2}}^{3}}+\frac{3 x_{2}^{2} S_{0}^{2}}{8 \sigma_{z_{2}}^{5}}\right] \sigma^{5} \\
+\left[\frac{105 x_{2}^{2} \sigma_{z_{2}}}{128 \pi}+\left(\frac{9 x_{1} x_{2} \zeta^{2}}{8 \pi}-\frac{15 x_{1} x_{2}}{16 \pi}-\frac{15 x_{1}^{2} \lambda}{32 \pi}\right) \frac{1}{\sigma_{z_{2}}}\right. \\
\left.+\frac{3}{16 \pi \sigma_{z_{2}}^{3}}\left(-3 x_{1} x_{2} \zeta S_{0}+\lambda+\frac{x_{1}^{2}}{2}\right)+\frac{9 x_{1} x_{2} S_{0}^{2}}{128 \pi \sigma_{z_{2}}^{5}}\right] \sigma^{3} \\
+\left[\frac{15 x_{1} x_{2} \sigma_{z_{2}}}{32 \pi}+\left(\frac{3}{8 \pi} x_{1} \zeta^{2}-\frac{3 \lambda}{16 \pi}-\frac{3 x_{1}^{2}}{16 \pi}\right) \frac{1}{\sigma_{z_{2}}}+\left(\frac{1}{16 \pi}-\frac{3 x_{1}}{16 \pi} \zeta S_{0}\right) \frac{1}{\sigma_{z_{2}}^{3}}+\frac{3 x_{1} S_{0}^{2}}{128 \pi \sigma_{z_{2}}^{5}}\right] \sigma \\
+\left[\frac{3 x_{1}^{2} \sigma_{z_{2}}}{32 \pi}+\left(\frac{3}{4 \pi} \zeta^{2}-\frac{1}{8 \pi}\right) \frac{1}{\sigma_{z_{2}}}-\frac{3 \zeta S_{0}}{8 \pi \sigma_{z_{2}}^{3}}+\frac{3 S_{0}^{2}}{64 \pi \sigma_{z_{2}}^{5}}\right] \frac{1}{\sigma}+\frac{\sigma_{z_{2}}}{16 \pi \sigma^{3}}
\end{aligned}
$$

To minimize the criterion, let $\partial \min _{\sigma}\left\|L_{n l}(z) p_{l i n}(z \mid \sigma)\right\|_{\Re^{2}(\mu)}^{2} / \partial \sigma=0$, then $\sigma$ can be obtained numerically.

Figure 6 shows the MSA curves of the QZS vibration isolator using different analytical methods. The exact solutions can be determined by integrating Eq. (4.4) directly using the numerical method combined with Eq. (3.11). It can be seen that both the equivalent linearization method and the probabilistic linearization method overestimate the MSA. The weighted probabilistic linearization method gives better results with the numerical results than the other two analytical methods.

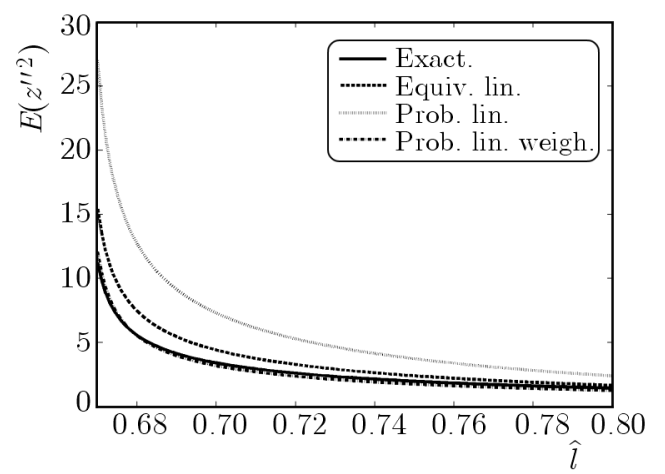

Fig. 6. MSA curves of the QZS vibration isolator using different analytical methods

$$
\left(\zeta=0.02, S_{0}=0.05\right)
$$

The MSA curves of the QZS vibration isolator for different values of damping ratio and spectral density of the random excitation using the weighted probabilistic linearization method 
are shown in Fig. 7. The MSA curves of the equivalent linear vibration isolator are also plotted in the same figure for comparison, which are plotted in the thinner lines. The MSA of the equivalent linear vibration isolator is given as

$$
E\left(z^{\prime \prime 2}{ }_{l}^{2}\right)=\left(1+4 \zeta_{l}^{2}\right) \sigma_{z_{l}}^{2}=\left(1+4 \alpha \zeta^{2}\right) \frac{S_{0}}{4 \sqrt{\alpha} \zeta}
$$

It is more complicated for the MSA case. With an increase in $\zeta$ or a decrease in $S_{0}$, the MSA of both vibration isolators decrease. For smaller values of $\zeta$, the MSA of the QZS vibration isolator is larger than the linear one, which indicates the isolation performance of the QZS vibration isolator is inferior to the linear one; when $\zeta$ continues to increase, the MSA is more or less the same for both vibration isolators; when $\zeta$ reaches a higher value, the MSA of the QZS vibration isolator is smaller than the linear one, which indicates that the QZS vibration isolator can achieve a better isolation performance. For smaller values of $S_{0}$, the MSA of the QZS vibration isolator is smaller than the linear one; when $S_{0}$ continues to increase, the MSA is more or less the same for both vibration isolators; when $S_{0}$ reaches a higher value, the MSA of the QZS vibration isolator is larger than the linear one, then the isolation performance of the QZS vibration isolator is inferior to the linear one. Also when the length ratio $\hat{l}$ increases, the MSA of both vibration isolators decrease.

(a)

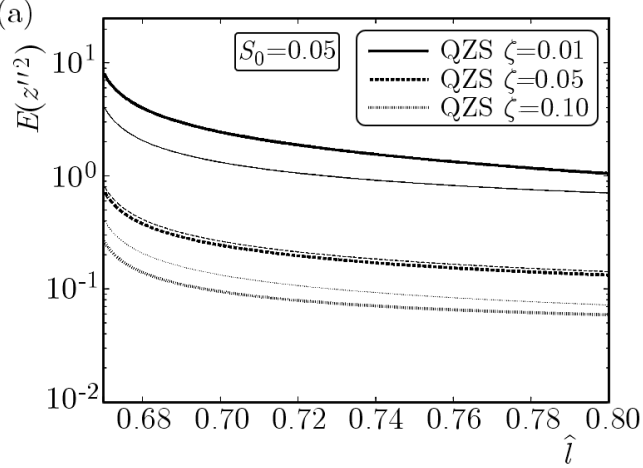

(b)

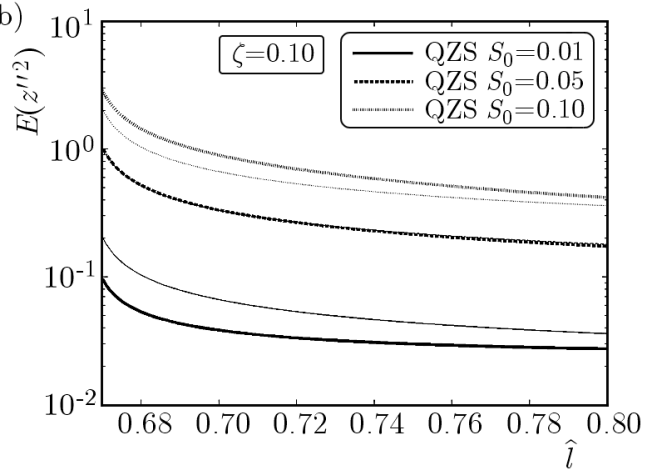

Fig. 7. MSA curves of the QZS vibration isolator for different values of damping ratio and spectral density of the random excitation

\section{Conclusions}

In this paper, the dynamic response and isolation performance of the QZS vibration isolator under random excitation are investigated. The QZS vibration isolator is comprised of a vertical spring providing positive stiffness and inclined springs used as negative stiffness correctors. The probability density function of the relative displacement of the mass is obtained by using probabilistic linearization method, and compared with the exact solution based on the FPK equation and the equivalent linearization method. The compared results show that both the probabilistic linearization method and equivalent linearization method give very good results in the tails of the probability density function, but the errors become larger in the peak value areas of the probability density function and the probabilistic linearization method gives better results than the equivalent linearization method. When the length ratio increases, which indicates that the nonlinear parameter $\lambda$ decreases, the errors of both methods become smaller in the peak value areas of the probability density function.

Two performance indexes (MSRD and MSA) are defined to evaluate the isolation performance of the QZS vibration isolator. The weighted probabilistic linearization method is employed to improve the accurate results of the two performance indexes by adding a weighted function to 
the criterion. The two performance indexes obtained by the weighted probabilistic linearization method are also compared with the numerical method, the equivalent linearization method and the probabilistic linearization method. The weighted probabilistic linearization method gives better results with the numerical results than the other two analytical methods.

The isolation performance of the QZS vibration isolator is also compared with an equivalent linear vibration isolator. The MSRD of the QZS vibration isolator is always lower than the equivalent linear vibration isolator, which indicates that the QZS vibration isolator can achieve a better isolation performance for this case. The MSA of the QZS vibration isolator can be larger or lower than the equivalent linear vibration isolator based on the values of damping ratio and spectral density of the random excitation, which is different from the MSRD case. For the MSA case, the spectral density of the random excitation should be considered first, and choose an appropiate damping ratio for the QZS vibration isolator to provide a better random isolation performance than the equivalent linear vibration isolator.

\section{Acknowledgments}

This work was supported by China Scholarship Council (CSC) and also supported by Funding of Jiangsu Innovation Program for Graduate Education (grant number KYLX_0243) and the Fundamental Research Funds for the Central Universities.

\section{References}

1. Alabuzhev P., Gritchin A., Kim L., Migirenko G., Chon V. And Stepanov P., 1989, Vibration Protecting and Measuring Systems with Quasi-Zero Stiffness, Hemisphere Publishing, Taylor \& Francis Group, New York

2. CAI G.Q., Lin Y.K., 1988, A new approximate solution technique for randomly excited non-linear oscillators, International Journal of Non-Linear Mechanics, 23, 5, 409-420

3. Carrell A., Brennan M.J., Waters T.P., 2007, Static analysis of a passive vibration isolation with quasi zero-stiffness characteristic, Journal of Sound and Vibration, 301, 3, 678-689

4. Caughey T.K., 1963, Equivalent linearization techniques, The Journal of the Acoustical Society of America, 35, 11, 1706-1711

5. Elishakoff I., Cai G.Q., 1993, Approximate solution for nonlinear random vibration problems by partial stochastic linearization, Probabilistic Engineering Mechanics, 8, 3, 233-237

6. Hao Z., CaO Q., 2014, A novel dynamical model for GVT nonlinear supporting system with stable-quasi-zero-stiffness, Journal of Theoretical and Applied Mechanics, 52, 1, 199-213

7. Harris C.M., Piersol A.G., 2002, Shock and Vibration Handbook, McGraw-Hill, New York

8. Ibrahim R.A., 2008, Recent advances in nonlinear passive vibration isolators, Journal of Sound and Vibration, 314, 3, 371-452

9. KIRK C.L., 1988, Non-linear random vibration isolators, Journal of Sound and Vibration, 124, 1, $157-182$

10. Klein G.H., 1964, Random excitation of a nonlinear system with tangent elasticity characteristics, The Journal of the Acoustical Society of America, 36, 11, 2095-2105

11. Kovacic I., Brennan M.J., Waters T.P., 2008, A study of a nonlinear vibration isolator with a quasi-zero stiffness characteristic, Journal of Sound and Vibration, 315, 3, 700-711

12. Le T.D., Ahn K.K., 2011, A vibration isolation system in low frequency excitation region using negative stiffness structure for vehicle seat, Journal of Sound and Vibration, 330, 26, 6311-6335

13. Lin Y.K., 1967, Probabilistic Theory of Structural Dynamics, McGraw-Hill, New York 
14. Liu X., Huang X., Hua H., 2013, On the characteristics of a quasi-zero stiffness isolator using Euler buckled beam as negative stiffness corrector, Journal of Sound and Vibration, 332, 14, $3359-3376$

15. Liu X., Huang X., Hua H., 2014, Performance of a zero stiffness isolator under shock excitations, Journal of Vibration and Control, 20, 14, 2090-2099

16. LYON R.H., 1960, On the vibration statistics of a randomly excited hard-spring oscillator, The Journal of the Acoustical Society of America, 32, 6, 716-719

17. LYON R.H., 1961, On the vibration statistics of a randomly excited hard-spring oscillator II, The Journal of the Acoustical Society of America, 33, 10, 1395-1403

18. Polidori D.C., Beck J.L., 1996, Approximate solutions for non-linear random vibration problems, Probabilistic Engineering Mechanics, 11, 3, 179-185

19. Polidori D.C., Beck J.L., Papadimitriou C., 2000, A new stationary PDF approximation for non-linear oscillators, International Journal of Non-linear Mechanics, 35, 4, 657-673

20. Robertson W.S., Kinder M.R.F., Cazzolato B.S., Zander A.C., 2009, Theoretical design parameters for a quasi-zero stiffness magnetic spring for vibration isolation, Journal of Sound and Vibration, 326, 1/2, 88-103

21. Shaw A.D., Neild S.A., WAGG D.J., 2013, A nonlinear spring mechanism incorporating a bistable composite plate for vibration isolation, Journal of Sound and Vibration, 332, 24, 6265-6275

22. SHIN K., 2014, Experimental investigation of the vibration transmissibility of a magnet-spring vibration isolator under random excitation, Journal of Vibroengineering, 16, 4, 1745-1752

23. WAng Y., Li S.M., Li J.Y., 2014, Response and performance of a nonlinear vibration isolator with high-static-low-dynamic-stiffness under shock excitations, Journal of Vibroengineering, 16, 7, $3382-3398$

24. WU W.F., LiN Y.K., 1984, Cumulant-neglect closure for non-linear oscillators under random parametric and external excitations, International Journal of Non-Linear Mechanics, 19, 4, 349-362

25. Xu D., Yu Q., Zhou J., 2013, Theoretical and experimental analyses of a nonlinear magnetic vibration isolator with quasi-zero-stiffness characteristic, Journal of Sound and Vibration, 332, 14, $3377-3389$

26. Zhou N., Liu K., 2010, A tunable high-static low-dynamic stiffness vibration isolator, Journal of Sound and Vibration, 329, 9, 1254-1273 